\title{
ANÁLISE DA REPRODUTIBILIDADE DO PROCESSO DE FABRICAÇÃO DE CÉLULAS SOLARES DE CIGS*
}

\author{
Paulo Victor Nogueira da Costa ${ }^{1}$ \\ Matheus Garcia Fonseca ${ }^{2}$ \\ Renan de Melo Correia Lima ${ }^{3}$ \\ Rodrigo Amaral de Medeiro ${ }^{4}$ \\ E. Schneller ${ }^{5}$ \\ Neelkanth G. Dhere ${ }^{6}$ \\ O. Shinde \\ Carlos Luiz Ferreira ${ }^{8}$ \\ Alaelson Vieira Gomes ${ }^{9}$ \\ Leila Rosa $\mathrm{Cruz}^{10}$
}

\section{Resumo}

Este trabalho visa analisar a reprodutibilidade do processo de produção de células solares de disseleneto de cobre índio e gálio (CIGS). O trabalho faz parte de um programa de colaboração entre o Instituto Militar de Engenharia (IME) e o Florida Solar Energy Center (FSEC). As células foram parcialmente produzidas no FSEC e finalizadas no IME. No FSEC foi fabricada a estrutura substrato/contato/CIGS/CdS, enquanto no IME foi produzida a estrutura do contato frontal, que consistia de uma bicamada de $\mathrm{ZnO} / \mathrm{ZnO}: \mathrm{Al}$ sob uma grade metálica de $\mathrm{Cr} / \mathrm{Ag}$. A caracterização foi feita através das curvas corrente $(\mathrm{I}) \mathrm{x}$ tensão $(\mathrm{V})$. A análise de reprodutibilidade foi feita de duas formas: experimento fatorial e diferença mínima significante (d.m.s) onde se utilizou o teste de Tukey. Com exceção da eficiência solar, os resultados mostraram uma dispersão nas médias dos parâmetros fotovoltaicos nos diferentes substratos, os quais dependiam da fornada de produção, mas demostraram uma homogeneidade nas propriedades das células produzidas ao longo dos substratos.

Palavras-chave:Célula solar; CIGS; análise estatística; reprodutibilidade

\section{REPRODUCIBILITY ANALISYS OF CIGS SOLAR CELLS PROCESSING Abstract}

The objective of this work is to analyze the reproducibility of the fabrication process of copper-indium-gallium diselenide solar cells. The work is part of a collaborative program between the Military Institute of Engineering (IME) and the Florida Solar Energy Center (FSEC). The solar cells were partially produced at FSEC and finished at IME. The substrate/contact/CIGS/CdS structure was fabricated at FSEC, while the the front contact structure consisting of a $\mathrm{ZnO} / \mathrm{ZnO}$ : Al bilayer and a $\mathrm{Cr} / \mathrm{Ag}$ metallic grid was prepared at IME. The characterization consisted in obtaining the current $(\mathrm{I}) \mathrm{X}$ voltage (V) curves. The reproducibility analysis was done using two tests: factorial experiment and minimum significant difference. Except for the efficiency, the results showed a dispersion in the photovoltaic parameters at different substrates, which depended on the production batch, but showed that the properties along each substrate were uniform.

Keywords: Solar cell; CIGS; statistical analysis; reproducibility.

1 Física, Bacharel em Física, Aluno de Mestrado, Seção de Engenharia Mecânica e de Materiais, Instituto Militar de Engenharia (IME), Rio de Janeiro, RJ e Brasil

2 Engenharia Elétrica, Aluno de Graduação, Seção de Engenharia Mecânica e de Materiais, Instituto Militar de Engenharia (IME), Rio de Janeiro, RJ e Brasil 
3 Construção Naval, Mestre, Aluno de Doutorado, Departamento de Engenharia Química e de Materiais, Pontifícia Universidade Católica, Rio de Janeiro, RJ e Brasil

4 Física, Doutor em Ciências, Pós Doc, Seção de Engenharia Mecânica e de Materiais, Instituto Militar de Engenharia (IME), Rio de Janeiro, RJ e Brasil

5 Física, PhD, scientist, FSEC, University of Central Florida, Cocoa, Florida, USA.

6 Física, PhD, Program Director, FSEC, University of Central Florida, Cocoa, Florida, USA.

7 Física, PhD, scientist, FSEC, University of Central Florida, Cocoa, Florida, USA.

8 Física, Doutor em Ciências, Professor, Seção de Engenharia Mecânica e de Materiais, Instituto Militar de Engenharia (IME), Rio de Janeiro, RJ e Brasil

9 Engenharia Metalúrgica, Doutor em Ciências, Professor, Seção de Engenharia Mecânica e de Materiais, Instituto Militar de Engenharia (IME), Rio de Janeiro, RJ e Brasil.

10 Física, Doutor em Ciências, Professor, Seção de Engenharia Mecânica e de Materiais, Instituto Militar de Engenharia (IME), Rio de Janeiro, RJ e Brasil 


\section{INTRODUÇÃO}

A energia solar fotovoltaica hoje é parte integrante da matriz elétrica de vários países, por exemplo, a China e a Alemanha [1-2]. Este mercado é basicamente dominado pelos módulos de silício cristalino, os módulos de filme fino representam apenas $8 \%$ do mercado [1-2]. No entanto, a eficiência das células de filme fino já alcançou a das células de silício multicristalino (24,1\%) [1-2], o que justifica investimentos e estudos nesta área.

As células mais investigadas de filme fino são as de silício amorfo [3-6], telureto de cádmio [3-6] e disseleneto de cobre e índio (CIS) ou disseleneto de cobre, índio e gálio (CIGS) [3-6].

O interesse pelas ligas de cobre para aplicação em células solares data de 1970. Três dessas ligas tiveram mais atenção, pois apresentavam uma banda proibida direta e podiam ser produzidas tipo-n ou tipo-p, com uma ampla gama de propriedades eletro-ópticas. Dentre essas ligas, o CIS foi a que mais se desenvolveu, pois possui um dos maiores coeficientes de absorção, suas propriedades eletro-ópticas são extremamente estáveis e sua banda proibida está próxima da banda ótima para eficiência máxima teórica [4-6]. Com a intenção de aumentar a banda proibida e, consequentemente aumentar a tensão de circuito aberto (Voc) da célula, é adicionado gálio, que entra no sítio do índio, utilizando-se uma concentração entre $25-30 \%$ de gálio, a banda aumenta de 1,02 eV para 1,15$1,20 \mathrm{eV}$ [4]. Com uma maior concentração de $\mathrm{Ga}(>30 \%)$, as células fornecem um maior Voc [4], mas sua eficiência é reduzida, pois menos do espectro solar é absorvido, ocasionando uma menor corrente fotogerada e consequentemente uma menor eficiência.

Uma célula típica de CIGS é depositada na configuração chamada de "substrato" (substrato/contato traseiro/CIGS/CdS/contato frontal), pois verificou-se que as células baseadas em superstratos têm uma eficiência inferior comparada com as células de substratos [6]. Geralmente a célula de CIGS é formada por um contato traseiro de molibdênio (Mo) devido a sua natureza inerte, em seguida, deposita-se a camada absorvedora tipo-p de CIGS. A camada tipo-n de sulfeto de cádmio (CdS), chamada de janela da célula, é depositada por banho químico (CBD). Como a camada janela absorve os fótons mais energéticos (na faixa do azul), então, para melhorar a resposta da célula no azul (comprimento de onda em torno de 400nm) deve-se trabalhar com espessuras de CdS na faixa de 50-100 nm[6]. Após a deposição da camada de janela, adiciona-se o contado frontal, que geralmente é um óxido condutor transparente (TCO), um semicondutor do tipo-n degenerado com boa condutividade elétrica e alta transparência no espectro do visível. Para melhorar a coleta de portadores, uma grade metálica é usada sobre o TCO. Dispositivos de CIGS de alta eficiência são geralmente fabricados com estruturas de bicamadas [6].

A eficiência dos dispositivos de CIGS depende muito fortemente da composição da liga, a qual deve ser otimizada para obter o melhor compromisso entre alta densidade de corrente de curto circuito (Jsc) e alto Voc. Por isso, é fundamental o controle dos parâmetros de forma a garantir a reprodutibilidade do processo, desta forma, este trabalho visa analisar a reprodutibilidade do processo de produção de células solares de CIGS fabricadas conjuntamente no Instituto Militar de Engenharia (IME) e no Florida Solar Energy Center (FSEC). 


\section{MATERIAIS E MÉTODOS}

Os dispositivos solares foram depositados em substratos de vidro com $12,5 \mathrm{~cm}^{2} \mathrm{com}$ a seguinte configuração: vidro/Mo/CIGS/CdS/ZnO/ZnO:Al/metal. A camada de Mo, com espessura de $1 \mu \mathrm{m}$, seguida pela camada de uma liga CuGa e pela de índio (In), foram depositadas por pulverização catódica DC, as camadas de CuGa e In foram posteriormente selenizadas em uma mistura diluída de seleneto de dietil em nitrogênio em um forno convencional a $500^{\circ} \mathrm{C}$, para formar a camada de tipo-p do semicondutor de Culn ${ }_{1-x} \mathrm{Ga}_{x} \mathrm{Se}_{2}$ sobre a camada de Mo. A camada tipo-n da heterojunção, CdS, com espessura de 0,05 $\mu \mathrm{m}$, foi preparada por deposição por banho químico, em uma solução de acetato de cádmio, tiouréia e hidróxido de amônio em água deionizada a $60-80^{\circ} \mathrm{C}$. Todas estas etapas foram realizadas no FSEC. As células foram finalizadas no IME, uma bicamada transparente e condutora de $\mathrm{ZnO} / \mathrm{ZnO}: \mathrm{Al}$, com espessura de 0,4-0,6 $\mu \mathrm{m}$, foi depositada por pulverização catódica com rádio frequência, para atuar como contato frontal, além disso foi feita a deposição de uma grade de contato frontal de $\mathrm{Cr} / \mathrm{Ag}$ com espessura de 0,05/3 $\mu \mathrm{m}$, depositada por evaporação por feixe de elétrons.

Nove células foram isoladas ao longo de cada substrato, permitindo a caracterização de vários dispositivos fabricados a partir do mesmo processo. A área útil da célula é de aproximadamente $0,6 \mathrm{~cm}^{2}$. Foram caracterizadasas nove células solares de 3 substratos (I,II e III), todos fabricados com os mesmos parâmetros de deposição. Os parâmetros fotovoltaicos das células foram colhidos através das curvas I $\mathrm{x}$ V. Ambas as análises foram feitas no LFF-IME, um simulador solar montado no próprio LFF-IME foi utilizado, juntamente com uma célula referência, para gerar a condição de $1 \mathrm{sol}\left(100 \mathrm{~mW} / \mathrm{cm}^{2}\right)$.

Foram realizados estudos estatísticos para todos os parâmetros fotovoltaicos: eficiência $(\eta)$, tensão em circuito aberto (Voc), corrente (Isc) em curto circuito e fator de forma (FF). A análise de reprodutibilidade foi feita com dois testes: experimento fatorial e diferença mínima significante (d.m.s), onde, neste último, se utilizou o teste de Tukey [7]. Como a eficiência depende do FF, do Isc e do Voc (equação 1), então o experimento fatorial avalia se há diferenças ou alguma reprodução do processo. As resistências em paralelo (Rshunt) e série (Rs) também foram avaliadas.

As análises estatísticas foram divididas em dois grupos: i) um grupo de reprodutibilidade de processo, que é formado pelos 3 substratos (I, II e III), chamado de grupo S; ii) um grupo de posicionamento das células em cada substrato, chamado de $\mathrm{B}$, onde a homogeneidade das propriedades das nove células é avaliada ao longo dos substratos. Como há nove células em cada substrato (N1, N2, ..., N9), o grupo B foi dividido em três sub-grupos, com três células cada: 1 (N1, N2, N3), 2 (N4, N5, N6) e 3 (N7, N8, N9). Estes sub-grupos identificam a posição no substrato: borda superior (1), centro (2) e borda inferior (3). A Figura 1 mostra um esquema do sistema analisado.

Foi utilizada a ferramenta estatística Experimento Fatorial para avaliar se há diferenças entre os grupos $\mathrm{S}$ e $\mathrm{B}$, bem como se há interação entre estes grupos, com um nível de confiança de $95 \%$. 


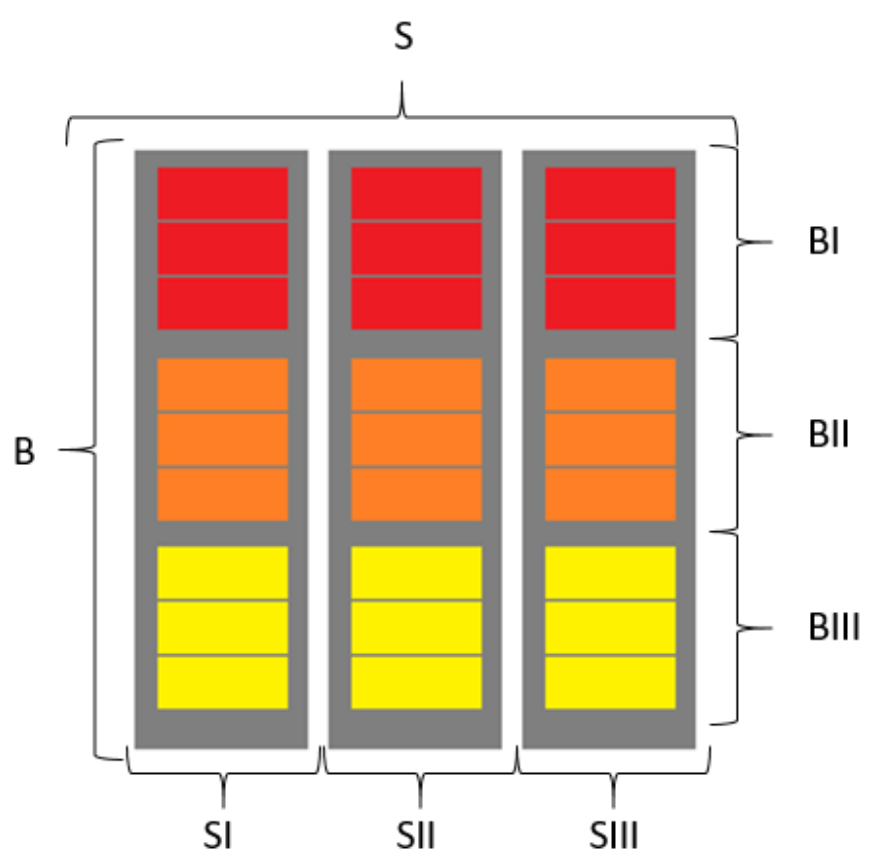

Figura 1. Esquema da divisão em grupos

O teste d.m.s realiza uma diferença de médias, em valor absoluto, e a compara com o valor crítico do teste de Tukey, equação 2. Com isso, determina-se o(s) grupo(s) que apresenta(m) o(s) valor(es) maior(es) para o parâmetro fotovoltaico, com um nível de confiança de $95 \%$. O teste d.m.s é mais indicado quando há um valor de $\mathrm{F}$ expressivo, permitindo identificar os grupos com médias significativamente diferentes, que contribuíram para o $\mathrm{F}$ calculado.

$$
\begin{aligned}
& \eta=\frac{F F V_{O C} I_{S C}}{P_{I N C}} \\
& \text { d.m.s }=q \sqrt{\frac{Q M R}{r}}, \text { ré o número de repetições }
\end{aligned}
$$

\section{RESULTADOS E DISCUSSÃO}

\subsection{PARÂMETROS FOTOVOLTAICOS}

As Tabelas 1, 2 e 3 mostram os parâmetros fotovoltaicos de cada uma das 9 células fabricadas nos substratos I, II e III. Observa-se uma flutuação dos valores entre os substratos e também ao longo de cada substrato, além disso, nota-se que os valores de eficiência estão bem abaixo do recorde global, que é de $22,6 \%$ [7]. Comparando-se os demais parâmetros com os respectivos recordes, Voc $=741 \mathrm{mV}$, $\mathrm{Jsc}=37,8 \mathrm{~mA} / \mathrm{cm}^{2}$ e $\mathrm{FF}=80,6 \%$ [7], verifica-se que a eficiência foi limitada basicamente pelo Voc e pelo FF. O baixo valor de Voc está relacionado à deficiência em gálio [8]. Já o valor de FF foi limitado pela baixa resistência shunt. Segundo a literatura, para uma perda de potência de $3 \%$, os valores de Rshunt deve estar abaixo de $0,5 \Omega$ e Rs deve ser de aproximadamente $500 \Omega$ [9]. 
Tabela 1.Parâmetros fotovoltaicos das células CIGS crescidas no substrato I

\begin{tabular}{llllllll} 
Área $\left(\mathrm{cm}^{2}\right)$ & Voc $(\mathrm{V})$ & Isc $(\mathrm{mA})$ & $\mathrm{Jsc}\left(\mathrm{mA} / \mathrm{cm}^{2}\right)$ & $\mathrm{FF}(\%)$ & $\eta(\%)$ & $\begin{array}{l}\text { Rshunt } \\
\left(\Omega . \mathrm{cm}^{2}\right)\end{array}$ & $\mathrm{Rs}\left(\Omega . \mathrm{cm}^{2}\right)$ \\
0,43 & 0,24 & 14,0 & 32,6 & 41,0 & 3,2 & 74 & 7,0 \\
\hline 0,52 & 0,35 & 14,0 & 26,7 & 44,9 & 4,2 & 140 & 8,4 \\
\hline 0,56 & 0,39 & 21,0 & 37,4 & 41,7 & 6,0 & 110 & 8,0 \\
0,48 & 0,39 & 17,4 & 36,0 & 42,9 & 6,1 & 150 & 9,1 \\
\hline 0,51 & 0,41 & 18,0 & 35,2 & 38,0 & 5,4 & 89 & 11,0 \\
0,54 & 0,40 & 14,9 & 27,8 & 37,7 & 4,2 & 97 & 13,0 \\
0,54 & 0,40 & 14,8 & 27,8 & 36,3 & 4,0 & 80 & 15,0 \\
0,54 & 0,42 & 15,0 & 27,8 & 32,8 & 3,8 & 67 & 19,0 \\
0,52 & 0,33 & 12,8 & 24,5 & 42,2 & 3,4 & 94 & 11,0 \\
\hline
\end{tabular}

Tabela 2.Parâmetros fotovoltaicos das células CIGS crescidas no substrato II

\begin{tabular}{lllllllll}
\hline & Área $\left(\mathrm{cm}^{2}\right)$ & Voc $(\mathrm{V})$ & Isc $(\mathrm{mA})$ & $\mathrm{Jsc}\left(\mathrm{mA} / \mathrm{cm}^{2}\right)$ & $\mathrm{FF}(\%)$ & $\eta(\%)$ & $\begin{array}{l}\text { Rshunt } \\
\left(\Omega . \mathrm{cm}^{2}\right)\end{array}$ & $\mathrm{Rs}\left(\Omega . \mathrm{cm}^{2}\right)$ \\
\hline N1 & 0,65 & 0,31 & 17,2 & 26,5 & 44,5 & 3,7 & 120 & 6,5 \\
\hline N2 & 0,62 & 0,30 & 21,5 & 34,3 & 43,6 & 4,5 & 170 & 5,6 \\
\hline N3 & 0,75 & 0,31 & 25,9 & 35,7 & 41,7 & 4,6 & 90 & 5,2 \\
\hline N4 & 0,68 & 0,18 & 23,9 & 35,4 & 43,2 & 4,8 & 99 & 5,3 \\
\hline N5 & 0,68 & 0,32 & 24,0 & 35,5 & 43,0 & 4,8 & 88 & 5,2 \\
\hline N6 & 0,65 & 0,32 & 18,8 & 28,7 & 46,3 & 4,2 & 170 & 5,8 \\
\hline N7 & 0,55 & 0,33 & 19,5 & 35,5 & 46,7 & 5,5 & 140 & 5,5 \\
\hline N8 & 0,62 & 0,33 & 21,6 & 34,5 & 45,2 & 5,2 & 130 & 5,3 \\
\hline N9 & 0,61 & 0,33 & 17,3 & 28,2 & 47,4 & 4,4 & 280 & 6,0 \\
\hline
\end{tabular}

Tabela 3.Parâmetros fotovoltaicos das células CIGS crescidas no substrato III

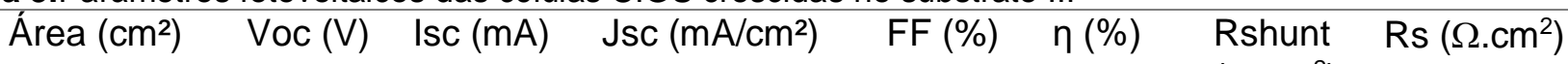

\begin{tabular}{lllllllll} 
N1 & 0,52 & 0,31 & 16,8 & 32,2 & 38,9 & 3,9 & 61 & 8,1 \\
\hline N2 & 0,42 & 0,30 & 17,7 & 42,2 & 32,7 & 4,2 & 34 & 10,0 \\
\hline N3 & 0,50 & 0,33 & 17,8 & 35,6 & 35,3 & 4,2 & 50 & 9,7 \\
\hline N4 & 0,52 & 0,34 & 18,8 & 36,1 & 34,1 & 4,2 & 38 & 10,0 \\
\hline N5 & 0,45 & 0,34 & 14,5 & 32,3 & 33,6 & 4,0 & 79 & 12,0 \\
\hline N6 & 0,50 & 0,34 & 17,7 & 35,4 & 37,5 & 4,5 & 64 & 9,4 \\
\hline N7 & 0,50 & 0,35 & 20,9 & 41,8 & 36,5 & 5,3 & 57 & 8,7 \\
\hline N8 & 0,55 & 0,35 & 21,9 & 39,1 & 36,1 & 4,9 & 53 & 8,8 \\
\hline N9 & 0,55 & 0,34 & 18,9 & 34,3 & 33,5 & 3,9 & 38 & 11,0 \\
\hline
\end{tabular}

\subsection{Experimento Fatorial}

As tabelas 4-9 mostram os resultados do experimento fatorial para os diversos parâmetros fotovoltaicos. Os valores obtidos de $\mathrm{F}$ foram comparados com Fcrit [10], com um nível de confiança de $95 \%$.

Observa-se que, para a eficiência, não há um número importante de $F$, para nenhuma das causas de variação, pois os valores de $F$ são menores do que o Fcrit, logo, a eficiência não depende do grupo de reprodutibilidade de processo (S) ou do grupo de homogeneidade (B). Então, as médias de eficiência são iguais em ambos os grupos, com confiança de $95 \%$.

Para o fator de forma, foi obtido um valor de $\mathrm{F}$ decisivo para o grupo S, ou seja, o processo de produção influenciou este fator de cada substrato, mostrando falta de reprodutibilidade. Para o grupo $B$ não há um $F$ significante, o que mostra que o valor 
de FF não depende da posição da célula no substrato. Para o tratamento, foi obtido um $\mathrm{F}$ expressivo, indicando que um dos grupos, $\mathrm{S}$ ou $\mathrm{B}$, é favorecido, confirmando a significância do $\mathrm{F}$ do grupo $\mathrm{S}$. Logo, não houve uma reprodução dos valores de $\mathrm{FF}$ para cada substrato, mas houve uma homogeneidade de FF ao longo de cada substrato. O mesmo comportamento foi observado para os parâmetros fotovoltaicos Voc, Isc e Rshunt, ou seja, um F significante foi obtido somente para o grupo S e para tratamentos, mostrados nas tabelas 6, 7 e 8 . Isto mostra que, para estes parâmetros, não houve uma reprodutibilidade no processo, mas houve uma homogeneidade ao longo dos substratos.

Para o Rs, o valor de F foi eloquente para os tratamentos, grupos S e B, sendo insignificante para as interações, então, para o Rs não houve reprodutibilidade no processo e nem homogeneidade ao longo dos substratos.

Tabela 4. Experimento fatorial para a eficiência das células.

\begin{tabular}{cccccc}
\hline $\begin{array}{c}\text { Causas de } \\
\text { Variação }\end{array}$ & GL & SQ & QM & F & Fcrit \\
\hline S & 2 & 0,42 & 0,21 & 0,43 & 3,55 \\
\hline B & 2 & 0,85 & 0,42 & 0,86 & 3,55 \\
\hline S X B & 4 & 3,15 & 0,79 & 1,42 & 2,73 \\
\hline Tratamentos & 8 & 4,42 & 0,55 & 1,12 & 2,51 \\
\hline Resíduo & 18 & 8,88 & 0,49 & & \\
\hline Total & 26 & 13,30 & & & \\
\hline
\end{tabular}

Tabela 5. Experimento fatorial para o FF das células.

\begin{tabular}{cccccc}
\hline $\begin{array}{c}\text { Causas de } \\
\text { Variação }\end{array}$ & GL & SQ & QM & F & Fcrit \\
\hline S & 2 & 389,69 & 194,84 & 29,39 & 3,55 \\
\hline B & 2 & 4,34 & 2,17 & 0,33 & 3,55 \\
\hline S x B & 4 & 56,31 & 14,08 & 0,25 & 2,73 \\
\hline Tratamentos & 8 & 450,33 & 56,29 & 8,49 & 2,51 \\
\hline Resíduo & 18 & 119,33 & 6,30 & & \\
\hline Total & 26 & 569,67 & & & \\
\hline
\end{tabular}

Tabela 6. Experimento fatorial para a Voc das células.

$\begin{array}{cccccc}\begin{array}{c}\text { Causas de } \\ \text { Variação }\end{array} & \text { GL } & \text { SQ } & \text { QM } & \text { F } & \text { Fcrit } \\ \text { S } & 2 & 0,020 & 0,010 & 5,77 & 3,55 \\ \text { B } & 2 & 0,006 & 0,003 & 1,83 & 3,55 \\ \text { S x B } & 4 & 0,010 & 0,002 & 0,54 & 2,73 \\ \text { Tratamentos } & 8 & 0,036 & 0,004 & 2,61 & 2,51 \\ \text { Resíduo } & 18 & 0,031 & 0,002 & & \\ \text { Total } & 26 & 0,067 & & & \end{array}$

Tabela 7. Experimento fatorial para a Isc das células.

\begin{tabular}{cccccc}
$\begin{array}{c}\text { Causas de } \\
\text { Variação }\end{array}$ & GL & SQ & QM & F & Fcrit \\
\hline S & 2 & 126,09 & 63,04 & 9,33 & 3,55 \\
\hline B & 2 & 1,55 & 0,78 & 0,11 & 3,55 \\
\hline S x B & 4 & 44,42 & 11,10 & 0,52 & 2,73 \\
\hline Tratamentos & 8 & 172,06 & 21,51 & 3,18 & 2,51 \\
\hline
\end{tabular}




$\begin{array}{clll}\text { Resíduo } & 18 & 121,63 & 6,76 \\ \text { Total } & 26 & 293,69 & \end{array}$

Tabela 8. Experimento fatorial para a Rshunt das células.

\begin{tabular}{cccccc}
$\begin{array}{c}\text { Causas de } \\
\text { Variação }\end{array}$ & GL & SQ & QM & F & Fcrit \\
\hline S & 2 & 36662,89 & 18331,44 & 11,99 & 3,55 \\
\hline B & 2 & 482,67 & 241,33 & 0,16 & 3,55 \\
\hline S x B & 4 & 9024,44 & 2256,11 & 0,39 & 2,73 \\
\hline Tratamentos & 8 & 4170,00 & 5771,25 & 3,78 & 2,51 \\
\hline Resíduo & 18 & 27512,67 & 1528,48 & & \\
\hline Total & 26 & 73682,67 & & & \\
\hline
\end{tabular}

Tabela 9. Experimento fatorial para a Rs das células.

\begin{tabular}{cccccc}
\hline $\begin{array}{c}\text { Causas de } \\
\text { Variação }\end{array}$ & GL & SQ & QM & F & Fcrit \\
\hline S & 2 & 155,29 & 77,65 & 27,31 & 3,55 \\
\hline B & 2 & 26,55 & 13,27 & 4,67 & 3,55 \\
\hline S x B & 4 & 54,08 & 13,52 & 0,46 & 2,73 \\
\hline Tratamentos & 8 & 235,91 & 29,49 & 10,37 & 2,51 \\
\hline Resíduo & 18 & 51,17 & 2,84 & & \\
\hline Total & 26 & 287,09 & & & \\
\hline
\end{tabular}

\subsection{Análise de d.m.s}

As tabelas 10-19 mostram os resultados da análise de d.m.s. Apenas quantidades com $\mathrm{F}$ significantes tiveram o valor de d.m.s calculado.

Como a eficiência, para todas as causas de variação, teve um $\mathrm{F}$ insignificante, então os valores das suas médias são iguais.

Para o FF, observa-se que há diferença entre as médias de SI, SII e SIII, conforme apresentado na Tabela 10. A análise de d.m.s., tal como indicou o valor de F no experimento fatorial, mostra que esta diferença é apreciável, pois elas são maiores que o valor de d.m.s. A média de SII é maior que a dos demais substratos, ou seja, as células do substrato SII apresentaram a maior média de FF. As médias dos tratamentos, apresentadas na Tabela 11 (analisando o grupo B dentro do grupo S), são iguais, mostrando que, para cada grupo $S$, os grupos $B$ são significativamente iguais, logo as propriedades das células são homogêneas. Para o Voc, verifica-se que a diferença entre as médias de SI e SII é aproximadamente igual a d.m.s, ou seja, a diferença é significante. A análise do tratamento forneceu uma d.m.s menor do que a calculada, mostrando que, para o Voc, pode-se considerar que houve uma homogeneidade dentro de cada substrato. Para o Isc, a d.m.s mostrou uma diferença significativa somente entre as médias SI e SII, sendo que a média de SII é maior que a de SI. Também se observa, para o grupo B e para o caso do tratamento, que a diferença entre as médias foi menor que a d.m.s, logo, as médias são significativamente iguais, confirmando a homogeneidade dos parâmetros fotovoltaicos ao longo do substrato.

Com o cálculo da d.m.s, verifica-se que, para o Rshunt, a média de SIl é significativamente maior que a de SIII, enquanto que as médias de SII e SI e de SI e SIII são iguais. Para o grupo B e para os tratamentos, as médias foram significativamente iguais, ou seja, valores de diferença entre as médias menores que 
o da d.m.s. Já para a Rs, a análise de d.m.s. informa que a média de SI é maior que a média de SII e que a de SIII é maior que a de SII. A média de B3 é maior que a média de B1. Para os tratamentos, a média de SIB3 é significativamente maior que a média de SIB1. Então, no substrato SI, o valor de Rs não foi homogêneo.

Tabela 10. Análise da d.m.s para o FF das células: $q=4,96$; d.m.s. $=4,25$

Média Diferença entre as Significância médias

das

diferenças

$\begin{array}{lllll}\text { SI } & 39,722 & \text { SI-SII } & 4,93 & \operatorname{sim} \\ \text { SII } & 44,656 & \text { SI-SIII } & 4,37 & \operatorname{sim} \\ \text { SIII } & 35,356 & \text { SII-SIII } & 9,30 & \operatorname{sim}\end{array}$

Tabela 11. Análise da d.m.s. para os tratamentos do FF da célula: $q=4,96$; d.m.s. $=7,37$

Média Diferença entre as Significância médias das

\begin{tabular}{ccccc} 
& & & & diferenças \\
\hline SIB1 & 42,53 & SIB1-SIB2 & 3,00 & não \\
\hline SIB2 & 39,53 & SIB1-SIB3 & 5,43 & não \\
\hline SIB3 & 37,10 & SIB2-SIB3 & 2,43 & não \\
\hline SIIB1 & 43,27 & SIIB1-SIIB2 & 1,00 & não \\
\hline SIIB2 & 44,27 & SIIB1-SIIB3 & 3,17 & não \\
\hline SIIB3 & 46,43 & SIIB2-SIIB3 & 2,17 & não \\
\hline SIIIB1 & 35,63 & SIIIB1-SIIIB2 & 0,57 & não \\
\hline SIIIB2 & 35,07 & SIIIB1-SIIIB3 & 0,27 & não \\
\hline SIIIB3 & 35,37 & SIIIB2-SIIIB3 & 0,30 & não \\
\hline
\end{tabular}

Tabela 12. Análise da d.m.s. para a Voc das células: $q=4,96$; d.m.s. $=0,068$

Média Diferença entre as Significância médias

das

diferenças

$\begin{array}{ccccc}\text { SI } & 0,369 & \text { SI-SII } & 0,066 & \text { sim } \\ \text { SII } & 0,303 & \text { SI-SIII } & 0,036 & \text { não } \\ \text { SIII } & 0,333 & \text { SII-SIII } & 0,029 & \text { não }\end{array}$

Tabela 13. Análise da d.m.s. para os tratamentos da Voc das células: $q=4,96 ;$ d.m.s. $=0,12$ Média Diferença entre as Significância médias das diferenças

\begin{tabular}{ccccc}
\hline SIB1 & 0,325 & SIB1-SIB2 & 0,074 & não \\
\hline SIB2 & 0,399 & SIB1-SIB3 & 0,058 & não \\
\hline SIB3 & 0,384 & SIB2-SIB3 & 0,015 & não \\
\hline SIIB1 & 0,308 & SIIB1-SIIB2 & 0,036 & não \\
\hline SIIB2 & 0,272 & SIIB1-SIIB3 & 0,022 & não \\
\hline SIIB3 & 0,330 & SIIB2-SIIB3 & 0,058 & não \\
\hline SIIIB1 & 0,313 & SIIIB1-SIIIB2 & 0,057 & não \\
\hline SIIIB2 & 0,340 & SIIIB1-SIIIB3 & 0,032 & não \\
\hline SIIIB3 & 0,345 & SIIIB2-SIIIB3 & 0,005 & não \\
\hline
\end{tabular}


Tabela 14. Análise da d.m.s. para a Isc das células: $q=4,96$; d.m.s. $=4,29$

Média Diferença entre as Significância

médias

das

diferenças

$\begin{array}{lllll}\text { SI } & 15,76 & \text { SI-SII } & 5,3 & \text { sim } \\ \text { SII } & 21,05 & \text { SI-SIII } & 2,6 & \text { não } \\ \text { SIII } & 18,33 & \text { SII-SIII } & 2,7 & \text { não }\end{array}$

Tabela 15. Análise da d.m.s. para os tratamentos da Iscdas célula: $q=4,96 ;$ d.m.s. $=7,44$

Média Diferença entre as Significância

médias

das

diferenças

\begin{tabular}{ccccc} 
SIB1 & 16,30 & SIB1-SIB2 & 0,47 & não \\
\hline SIB2 & 16,77 & SIB1-SIB3 & 2,11 & não \\
\hline SIB3 & 14,20 & SIB2-SIB3 & 2,58 & não \\
\hline SIIB1 & 21,54 & SIIB1-SIIB2 & 0,62 & não \\
SIIB2 & 22,16 & SIIB1-SIIB3 & 2,09 & não \\
SIIB3 & 19,45 & SIIB2-SIIB3 & 2,72 & não \\
SIIIB1 & 17,43 & SIIIB1-SIIIB2 & 0,41 & não \\
\hline SIIIB2 & 17,01 & SIIIB1-SIIIB3 & 3,13 & não \\
\hline SIIIB3 & 20,56 & SIIIB2-SIIIB3 & 3,54 & não
\end{tabular}

Tabela 16. Análise da d.m.s. para a Rshunt das células: $q=4,96$; d.m.s. $=64,64$

Média Diferença entre as Significância

médias

das

diferenças

$\begin{array}{ccccc}\text { SI } & 100,1 & \text { SI-SII } & 42,8 & \text { não } \\ \text { SII } & 142,9 & \text { SI-SIII } & 47,4 & \text { não } \\ \text { SIII } & 52,7 & \text { SII-SIII } & 90,2 & \text { sim }\end{array}$

Tabela 17. Análise da d.m.s. para os tratamentos da Rshunt da célula: $q=4,96$; d.m.s. $=111,96$

Média Diferença entre as Significância

médias

das

diferenças

\begin{tabular}{ccccc} 
SIB1 & 108,0 & SIB1-SIB2 & 4,0 & não \\
\hline SIB2 & 112,0 & SIB1-SIB3 & 21,7 & não \\
\hline SIB3 & 80,3 & SIB2-SIB3 & 31,7 & não \\
SIIB1 & 126,7 & SIIB1-SIIB2 & 8,0 & não \\
SIIB2 & 118,7 & SIIB1-SIIB3 & 56,7 & não \\
SIIB3 & 183,3 & SIIB2-SIIB3 & 64,7 & não \\
SIIIB1 & 48,3 & SIIIB1-SIIIB2 & 12,0 & não \\
\hline SIIIB2 & 60,3 & SIIIB1-SIIIB3 & 1,0 & não \\
\hline SIIIB3 & 49,3 & SIIIB2-SIIIB3 & 11,0 & não
\end{tabular}

Tabela 18. Análise da d.m.s. para a Vocslope das células: $q=4,96 ;$ d.m.s. $=2,78$

Média Diferença entre as Significância médias

das

diferenças

$\begin{array}{ccccc}\text { SI } & 11,28 & \text { SI-SII } & 5,68 & \text { sim } \\ \text { SII } & 5,60 & \text { SI-SIII } & 1,53 & \text { não }\end{array}$




\begin{tabular}{ccccc}
\hline SIII & 9,74 & SII-SIII & 4,14 & sim \\
\hline B1 & 7,61 & B1-B2 & 1,37 & não \\
\hline B2 & 8,98 & B1-B3 & 2,42 & sim \\
\hline B3 & 10,03 & B2-B3 & 1,06 & não \\
\hline
\end{tabular}

Tabela 19. Análise da d.m.s. para os tratamentos da Rsda célula: $q=4,96 ;$ d.m.s. $=4,80$

\begin{tabular}{ccccc}
\hline & Média & $\begin{array}{c}\text { Diferença entre as } \\
\text { médias }\end{array}$ & $\begin{array}{c}\text { Significância } \\
\text { das } \\
\text { diferenças }\end{array}$ \\
\hline SIB1 & 7,8 & SIB1-SIB2 & 3,23 & não \\
\hline SIB2 & 11,0 & SIB1-SIB3 & 7,20 & sim \\
\hline SIB3 & 15,0 & SIB2-SIB3 & 3,97 & não \\
\hline SIIB1 & 5,8 & SIIB1-SIIB2 & 0,33 & não \\
\hline SIIB2 & 5,4 & SIIB1-SIIB3 & 0,17 & não \\
\hline SIIB3 & 5,6 & SIIB2-SIIB3 & 0,17 & não \\
\hline SIIIB1 & 9,3 & SIIIB1-SIIIB2 & 1,20 & não \\
\hline SIIIB2 & 10,5 & SIIIB1-SIIIB3 & 0,23 & não \\
\hline SIIIB3 & 9,5 & SIIIB2-SIIIB3 & 0,97 & não \\
\hline
\end{tabular}

\subsection{Discussão}

O valor de Voc depende da composição do CIGS, no caso, da quantidade de $\mathrm{Ga}$, então, o respectivo valor considerável de $\mathrm{F}$ no grupo $\mathrm{S}$ informa que não há uma reprodutibilidade na produção do CIGS nos três substratos. No entanto, ao longo de cada substrato há uma homogeneidade na deposição, porque o $F$ não foi significante. O grupo SI parece ter uma maior quantidade de Ga que o SII, pois apresenta uma média maior de Voc.

Para o Isc, outro parâmetro que depende das propriedades do CIGS, não houve reprodutibilidade, semelhante ao resultado do Voc, corroborando a informação de que a produção da camada CIGS não foi igual para os três substratos. Isto se deve ao fato de o processamento ter sido feito em fornadas diferentes. Porém, semelhante ao Voc, a camada CIGS foi homogênea ao longo do substrato.

No caso do FF, como é uma deposição de multicamadas, sabe-se que ele é muito limitado pelas resistências, Rs e Rshunt, cujas médias não foram iguais para os três substratos, assim, para o $\mathrm{FF}$, a diferença entre as médias no grupo $\mathrm{S}$ pode ser atribuída à introdução destas resistências nas diferentes camadas, de forma não controlada. A Rshunt, por exemplo, é introduzida via inserções de buracos nos filmes, enquanto a Rs pode ser inserida de várias formas, sendo a contribuição dos contatos, a forma mais relevante. Logo, também para o FF, o processo não foi reprodutível nos três substratos, mas ao longo de cada substrato este valor foi o mesmo.

Apesar da diferença nas médias de FF, Isc e Voc entre os três substratos, observa-se que a eficiência não foi afetada por esta diferença, pois a eficiência é calculada através do produto desses parâmetros, onde a diferença pode ter sido anulada. 


\section{CONCLUSÃO}

O experimento fatorial e a análise de d.m.s mostraram que há uma diferença decisiva nos valores de FF, Isc e Voc, nos três substratos. Isto demonstra que, apesar de o processamento ter sido o mesmo para os substratos SI, SII e SIII, ele não foi suficientemente controlado para garantir as mesmas propriedades, no entanto, observou-se que a eficiência, que é o parâmetro relevante, não foi afetada por esta diferença. A posição da célula ao longo de cada substrato não influenciou os parâmetros fotovoltaicos responsáveis pela eficiência. Esses resultados auxiliam na otimização do processo, pois se tem médias maiores e menores, em função dos grupos de reprodutibilidade. Com eles pode-se entender em qual processo houve discrepância que acarretou nessas diferenças de média e minimizar os erros para maximizar os parâmetros desejados.

\section{Agradecimentos}

Este trabalho foi financiado pelo CNPq (proc. 401313/2014-0) e pela CAPES (Programa de Demanda Social).

\section{REFERÊNCIAS}

1 Warmuth, W., CFraunhofer ISE: PhotovoltaicsReport (2016) Updated: October 2016,

https://www.ise.fraunhofer.de/content/dam/ise/de/documents/publications/studie s/Photovoltaics-Report.pdf., Acessado em maio de 2017.

2 REN 21 - 2016, http://www.ren21.net/status-of-renewables/global-status-report/, Acessado em maio de 2017.

3 Martin A. Green. Thin-film solar cells: review of materials, Technologies and comercial status. J. MaterSci: MaterElectron. 2007 (18:S15-S19)

4 Lawrence L. Kazmerski. Photovoltaics: A review of cell and module Technologies. RenewableandSustainable Energy Reviews. 1997. Vol. 1 pp. $71-170$

5 Lawrence L. Kazmerski. Solar photovoltaics R\&D at the tipping point: A 2005 technology overview. JournalofElectronSpectroscopyandRelatedPhenomena. 2006. 150 pp. $105-135$

6 K.L. Chopra, P. D. Paulson, V. Dutta. Thin-Fllm Solar Cells: An Overview. 2004.Prog. Photovolt: Res. Appl.; 12: 69 - 92

7 Philip Jackson, et al. Effects of heavy alkali elements in $\mathrm{Cu}(\mathrm{In}, \mathrm{Ga}) \mathrm{Se}_{2}$ solar cells with efficiencies up to 22,6\%. Phys. Status Solidi RRL 10, № 8, 583-586 2016.

8 Renan de Melo Correia Lima, et al. Caracterização de células solares de filmes finos de CIGS. Revista Matéria. 2016

9 FAHRENBRUCH, A.L., BUBE, R.H., Fundamentals of Solar Cells, $1^{\text {st }}$ ed., New York, Academic Press, 1983.

10 VIEIRA, SÔNIA, Estatística Experimental, 2ª ed., São Paulo, Atlas, 1999 